\title{
Quantitative Aberration-Corrected STEM for Studies of Oxide Superlattices and Topological Defects in Layered Ferroelectrics
}

Albina Borisevich ${ }^{1}$, Rama Vasudevan ${ }^{1}$, Yu Zhou ${ }^{2}$, Kyle Kelley ${ }^{1}$, Donovan Leonard ${ }^{1}$, Summayya Kouser $^{3}$, Sabine Neumayer ${ }^{1}$, Peter Maksymovych ${ }^{1}$, Sokrates Pantelides ${ }^{3}$, Steven May ${ }^{2}$ and Nina Balke ${ }^{1}$

${ }^{1}$ Oak Ridge National Laboratory, Oak Ridge, Tennessee, United States, ${ }^{2}$ Drexel University, Philadelphia, Pennsylvania, United States, ${ }^{3}$ Vanderbilt University, Nashville, Tennessee, United States

Quantitative aberration-corrected scanning transmission electron microscopy (STEM) has been an indispensable tool in studies of perovskites, where it has been demonstrated that the relevant order parameters, such as lattice distances, cation displacements, and oxygen octahedral rotations can be examined with single-unit-cell resolution and connected to local and global materials properties [1]. Octahedral tilts in particular are being actively explored as a new means of engineering of thin film and interface properties. Recently, it has been demonstrated that tilt variations in a completely isovalent ( $\mathrm{La}, \mathrm{Ca}) \mathrm{MnO} 3 /(\mathrm{La}, \mathrm{Sr}) \mathrm{MnO} 3$ (100) heterostructure can be used to create magnetic property modulations [2]. While across (100) interface, only two tilt components are rigidly coupled, for (111) oriented multilayers all three tilt components will be coupled, suggesting an interesting case to explore octahedral tilt engineering. Fig.1 shows HAADF and ABF images of a (La,Sr)MnO3/LaFeO3 (LSMO/LFO) superlattices, as well as the octahedral tilt map and octahedral tilt profile derived from the ABF image. Unexpectedly, the tilt structure of LFO acquires a striped character indicative of effective superlattice of $\sim 15 \AA$ in the [111] direction. This behavior, while reproducibly detected in the experiment, has not been predicted in the first-principles study of the system [3]. The work is currently underway to determine if the boundary conditions of the calculation can be changed to allow for this structure to emerge. Advanced data analysis methods have further expanded the capabilities of local studies with STEM to analyses of spatial correlations and other statistical properties [4]. Recently, layered ferroelectrics of the thiophosphate family, such as Copper Indium Thiophosphate (CIPS), have garnered attention of researchers due to high potential for tunability and therefore a wealth of possible phase transitions [5]. However, the layer arrangement has not been previously examined in cross-section, even though defects in layer stacking can be expected to affect polarization and switching behavior. A representative HAADF image of the CIPS crystal in cross-section is shown in Fig. 2(a). Layers can be clearly distinguished; it is also clear that their stacking has defects. Inset in Fig. 2(a) shows the image diffractogram, with the $<002>$ reflection corresponding to the layer spacing highlighted by the red circles. Using Geometric Phase analysis (GPA) approach [6] with $<002>$ and one of the longitudinal reflections we can compute a set of strain component maps highlighting defect sites and estimate the associated strain; Fig. 2(b) shows map of the Eyy component. Defects of different polarity and with different number of nodes are observed, interspersed by relatively unstrained areas of 5-15 $\mathrm{nm}$. We can also explore spatial distribution of the [002] spacing across the image. If a k-means clustering algorithm is applied to the resulting data, three types of clusters with distinct average spacing can be identified, possibly correlating to three types of ferroelectric behavior predicted by theory (Fig. 2(c)). A theory-driven interpretation of this data is currently underway [7]. 


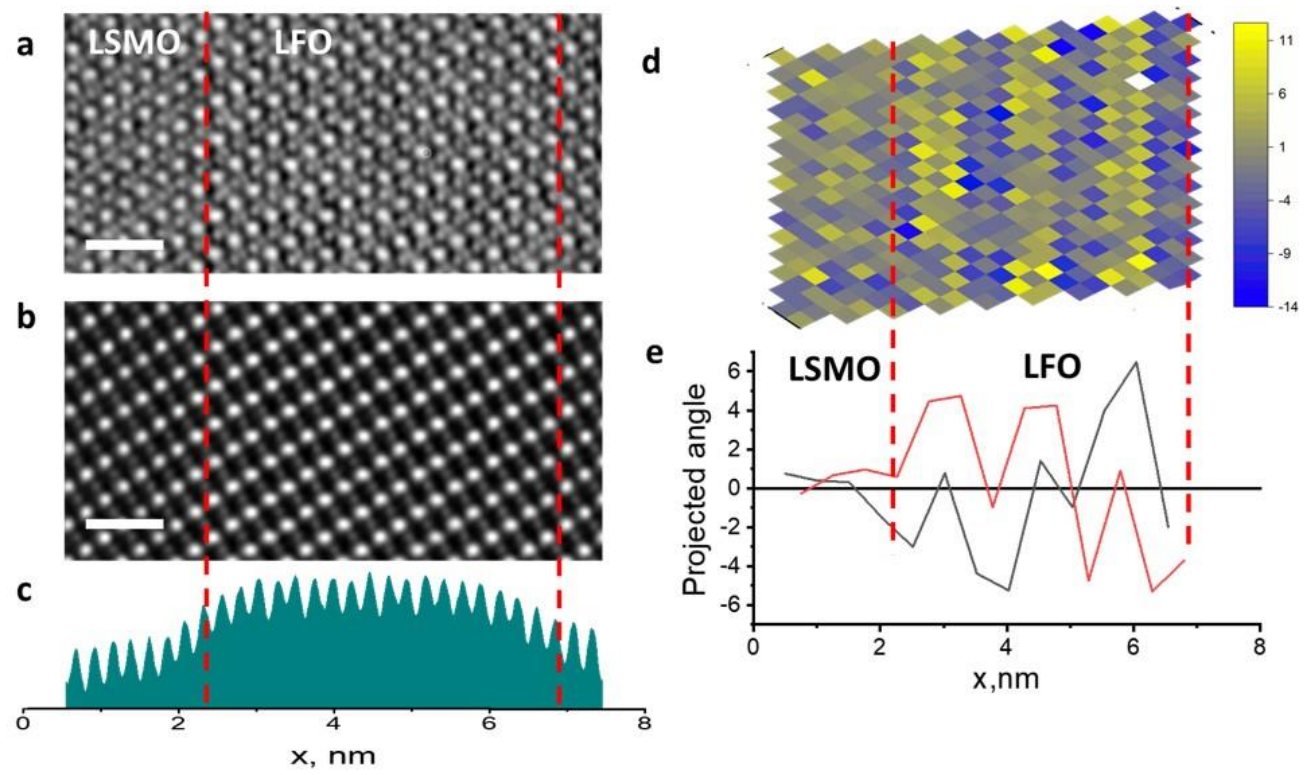

Figure 1. 1 Octahedral tilt behavior in LSMO/LFO superlattices grown on (111) STO: (a-b) Portions of a) Inverted $\mathrm{ABF}$ and $\mathrm{b}$ ) $\mathrm{HAADF}$ images of a region of the superlattice; c) intensity profile of the full corresponding HAADF image helping identify superlattice layers; (d) map of projected tilt angles computed from the same image. Tilt arrangement appears to be complex, but tilt amplitudes are increased inside LFO blocks. This increase is also evident in the tilt map profile (e); red dotted lines correspond to the location of interfaces, black and red lines correspond to averages of the alternating vertical lines in (d). Scale bars are $1 \mathrm{~nm}$. Image fragments in $(\mathrm{a}, \mathrm{b})$ have been lowpass filtered.
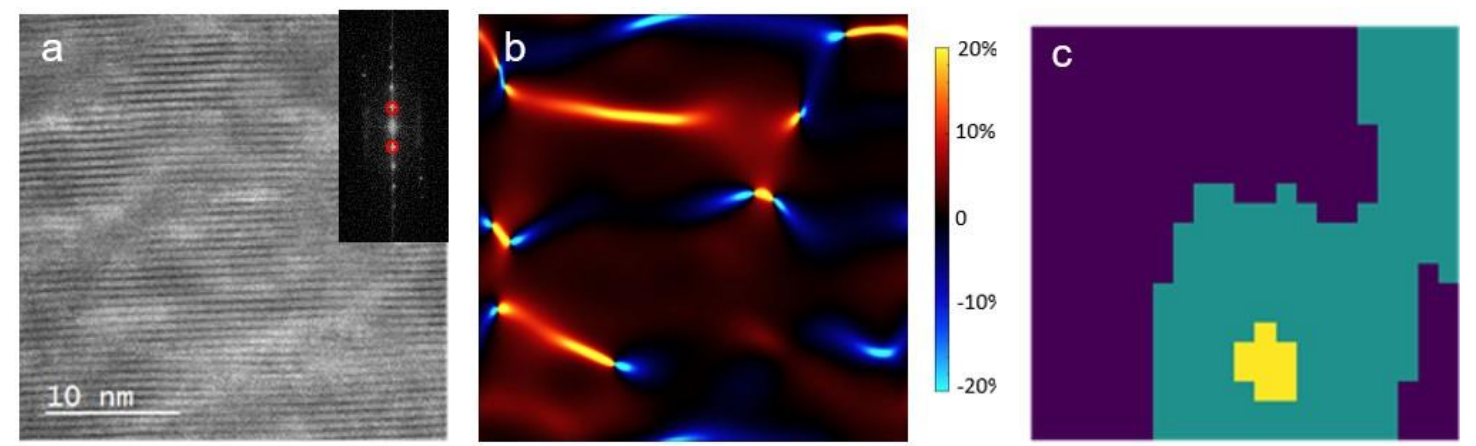

Figure 2. Defect analysis of CIPS cross-sectional images: (a) HAADF STEM image and the corresponding diffractogram (inset) with reflection highlighted, (c) strain component Eyy computed via GPA method and the corresponding color scale; (c) cluster map derived from (a) using local [002] spacings via k-means clustering.

\section{References}

[1] Ian MacLaren \& Quentin M. Ramasse, International Materials Reviews, 59, 115-131 (2014)

[2] E. J. Moon, et al., Phys. Rev. Lett. 119, 197204 (2017).

[3] Y. Zhou et al, Adv. Mater. Interfaces, in press (2020).

[4] A.Belianinov et al. Adv Struct Chem Imag, 1, 6 (2015).

[5] J.A Brehm et al., Nat. Mater., 19, 43 (2020).

[6] M. J. Hÿtch, E. Snoeck, and R. Kilaas, Ultramicroscopy 74, 131-146 (1998)

[7] Research was sponsored by the Division of Materials Science and Engineering, Basic Energy Sciences (BES), Office of Science, US Department of Energy (DOE). 